\title{
Joint hunt for missing particles
}

\section{Cosmologists and high-energy physicists, renowned for radical imaginativeness, seem to have infected solid-state physicists with enthusiasm for the search for dark matter.}

Astrophysicists and high-energy physicists alike are just now frustrated in their search for missing matter, the former because they have good reason to suppose matter to be present where they cannot see it, the latter because their descriptions require particles not yet found. The realization that both camps may after all be hunting the same entitities has already proved stimulating. It is even more enticing that condensed matter physics, pushed to new limits, may be cheaply used to find the elusive stuff.

Dark matter of one kind or another has been perplexing astrophysicists and cosmologists for some time. Evidence for its existence is still circumstantial - the anomalous orbital behaviour of stars in galaxies and, on a grander scale, theoretical prejudice (otherwise called the inflationary model of the very early Universe) that the mean density of the observable Universe should be about one hundred times that so far detected with visible or other radiation. Of the two principal candidates - light relativistic ('hot') neutrinos and more massive ('cold') particles - the second seems to fit the constraints better.

High-energy physics has its own dilemma. What is called the 'standard model', a combination of quantum chromodynamics (QCD), with its quark-gluon description of strongly interacting particles, and the gauge theory of electromagnetic and weak interactions, has made many successful predictions and is not contradicted by observation. But there remain some 20 free parameters' while the standard model requires the existence of new particles yet to be detected.

One such particle stems from what might be called a minor embarrassment of QCD. Unmodified, the theory would prefer the electric dipole moment of the neutron to be far greater than the tiny upper limit observed. To circumvent this difficulty, a new hidden symmetry has been imposed within the theory which, when broken at the appropriate energy, results in a massive particle called the axion.

This sounds arbitrary and aesthetically unsatisfying, but such shortcomings are outweighed by the explanatory success of QCD. Less experimentally compelling, but at least as appealing to their supporters (though, as the theorist John Ellis once remarked, mother aardvarks find their offspring appealing too), are the supersymmetry theories. Alas, their price for unifying three or all four of the funda- mental interactions is a plethora of putative particles.

Here is but one aspect of the crossfertilization of astronomy and high-energy physics: axions and supersymmetry particles have masses and other properties that make them particularly strong candidates for the cold dark matter, not only pervading the Universe as a whole but also gravitationally bound along with visible matter within galaxies. Galactic dark matter would be streaming through our laboratories but, to be detectable, would have to react sufficiently strongly with ordinary matter by means other than gravity.

Happily, it should. (See, for a review, P.F. Smith, Proceedings of the second CERN-ESO symposium, European Southern Observatory, 1986.) The axion, for example, should interact with a magnetic field to yield a photon. For the expected range of masses, an array of microwave cavities in a five-tesla field could be designed to detect the narrow signature of galactic axions somewhere in the very broad band between 1 and $1,000 \mathrm{GHz}$.

More widespread interest has been generated by the prospect of catching supersymmetry particles. Not only are the rewards potentially great, but the combination of disciplines involved is entertainingly unusual. In particular, solid state physicists are responding to the novel challenge of how to detect and identify the perturbation in a crystal lattice following the scattering off a nucleus of a dark matter particle. At low temperatures, the recoil of the nucleus generates quantized acoustic waves, or phonons, in the lattice. The trick is then either to detect the ensuing temperature rise or the phonons themselves.

The former is the approach adopted by Bernard Sadoulet and others at Berkeley and by Peter Smith and collaborators at the Rutherford Appleton Laboratory near Oxford. They take advantage of the exceedingly small heat capacity of tiny pure crystals at $10-20 \mathrm{mK}$ : a single $6 \mathrm{keV}$ photon can be detected by the 0.1 per cent temperature rise that it produces.

Different supersymmetry particles (sneutrinos, higgsinos and photinos seem to be the favourites) will give a variety of energy spectra according to the atomic mass and spin of the target nuclei, hence the planned simultaneous use of materials such as silicon, gallium arsenide and lithium bromide. Another signature is the seasonal variation in the particle spectrum caused by the Earth's orbital motion relative to the galactic flux. Such experiments will have to be conducted underground to ensure adequate shielding from cosmic rays.

There are other, perhaps more imaginative and even elegant, ways of detecting a nuclear recoil, such as that developed by A. Drukier and others at the Max Planck Institute at Munich. He proposes to suspend granules of superconducting material within a magnetic field, setting the temperature so that a recoil within a granule would flip it into its resistive state, causing a detectable movement of magnetic flux.

The temperature rise in such small detector crystals (typically of micron thickness) arises from the multiple scattering of phonons off surfaces. Nobody knows for sure what happens if, as might be desirable, the crystal size is increased. Lowenergy phonons, at least, experience little scattering within the crystal while the role of surface scattering would diminish, but nobody knows the energy spectrum of phonons generated by a recoil or how all the possible modes will propagate. Furthermore, the scattering properties of surfaces are very sensitive to the presence of small numbers of external atoms of contaminant.

With such uncertainties in mind, ambitious plans have been put forward to detect phonons directly. One being developed by Blas Cabrera and others at Stanford exploits the effect of phonons on a superconductor. If the phonons have sufficient energy they can disrupt the 'Cooper' pairs of electrons which, by means of their interactions with the lattice, otherwise flow freely. The disruption leaves a combination of holes and electrons which can in turn be detected by means of a tunnel junction.

What are the prospects? Even the furthest developed nuclear recoil detectors will take two to three years to become operational. Thereafter, supersymmetry particles may either be found immediately or, if the seasonal signal is required for convincing detection, only after a further year or two. But perhaps they will not be found at all. Agencies being asked to finance these shots in the dark would be right to take the gamble; gamblers elsewhere should bet on the last possibility, but if they are wrong, the excitement would surely compensate for the hole in their pocket.
Philip Campbell 